\title{
Why Replacing Different Oxygen of Thymine with Sulfur Causes Distinct Absorption and Intersystem Crossing?
}

\author{
Shuming Bai* and Mario Barbatti* \\ Aix Marseille Univ, CNRS, ICR, Marseille, France. \\ *E-mail addresses: shuming.bai@univ-amu.fr (SB); mario.barbatti@univ-amu.fr (MB); Tel : +33 (o)4 84529200
}

\begin{abstract}
Recent experiments replacing oxygen atoms by sulfur in thymine have revealed that absorption and intersystem crossings properties of these derivatives are strongly dependent on the position and number of the substitutions, affecting their potential performance for photodynamical therapy. Using multireference quantum chemical methods (CASPT2 and DFT/MRCI), we calculated absorption spectra and spin-orbit coupling matrix elements for thymine (Thy), 2thiothymine (2tThy), 4-thiothymine (4tThy), and 2,4-dithiothymine (2,4dtThy), to investigate this relation between structure and photophysics. The simulations showed that a simple 4-electrons/4-orbital minimum model can explain the main experimentally observed spectral features. Moreover, the computational estimate of intersystem crossing lifetimes in this sequence of molecules revealed that the experimental value attributed to thymine in water might be underestimated by a factor 20, most probably due to an overlap of singlet/triplet absorption signals in the transient absorption spectrum. The difference between the absorptivity of 2 tThy and 2 tThd was also investigated, but no conclusive explanation could be found.
\end{abstract}

\section{INTRODUCTION}

Photoinduced processes in nucleic acids ${ }^{1-4}$ is a very active area of research and the particular sub-field of photoprocesses in thio-modified nucleobases and nucleosides has become an important investigation topic. ${ }^{5,6}$ Besides its fundamental interest, 7 two practical reasons stimulating the research of photoinduced processes in thio-derivatives have been their carcinogenic role in patients taking these substances as immunosuppresants ${ }^{8-10}$ and their potential use as a chemotherapeutic agents. ${ }^{11}{ }^{12}$ In both cases, the underlying photophysical processes rely on the combination of the natural affinity of these derivatives to bind DNA and the capacity of their photoinduced triplet states of reacting with genetic material (usually via singlet oxygen generation), inducing mutations or killing the cell. Thus, either aiming at maximizing or minimizing the photoactivity of thio-derivatives, there are a number of photophysical properties to be optimized depending on the specific application, such as the absorption wavelength of these molecules, their absorptivity, their triplet quantum yields, and their quantum yields of singlet oxygen formation.

In this work, we focus on the photophysics of the thiothymines shown in Figure 1, which are obtained by systematic sulfur replacement at positions 2 and 4 . The basic physicalchemical properties of these systems and of the closely related thiouracils have been under scrutiny of experimentalists ${ }^{7,13-20}$ and theoreticians ${ }^{21-28}$ in the last years, especially after Harada and co-workers, ${ }^{17}$ based on pump-probe transient absorption spectroscopy, showed that the triplet formation in 4 tThd in water is complete in less than $10 \mathrm{ps,}$ with quantum yield near unity. This finding unveiled a completely distinct photophysics as compared to that of Thd, which, under the same conditions, converts to the singlet ground state through internal conversion within 0.5 ps. ${ }^{17}$ Even more astonishingly, Reichardt and Crespo-Hernandez ${ }^{19}$ determined that the triplet manifold of 4 tThd in water starts to be populated in the sub-picosecond scale, a remarkably fast intersystem crossing (ISC) process. This same ultrafast ISC was also observed in an aprotic solvent - acetonitrile - where 2tThy triplet formation occurs within $0.3 \mathrm{ps}$, with efficiency also approaching unity. ${ }^{15}$
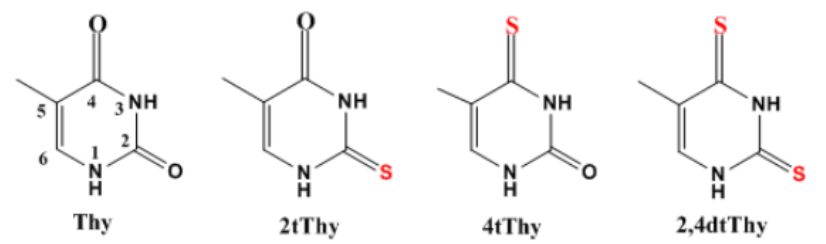

Figure 1. Structure of the molecules investigated in this paper: 2-thiothymine (2tThy), 4-thiothymine (4tThy), and 2,4dithiothymine (2,4dtThy). Throughout the text, equivalent notation will be used for thymidine (Thd) and uracil (Ura).

In spite of the high triplet quantum yield of single-substituted thiothymines, Kuramochi et al. ${ }^{18}$ showed that the quantum yield for singlet oxygen formation for 2 tThy in oxygen-saturated acetonitrile was rather modest, only 0.36 . This value, slightly increases to 0.46 for $2,4 \mathrm{dtThy}, 7$ and doubles to 0.69 upon aza-substitution at position 6 of 2tThy. ${ }^{18}$ 
Much of the research on the photophysics of thio-derivatives has been focused on determining the nature of the reaction pathways populating the triplet manifold. Based on absorption and emission measurements, Harada et al. ${ }^{20}$ proposed that the ISC in 4 tThd in acetonitrile should occur through a $\mathrm{S}_{1}\left({ }^{1} \mathrm{n} \pi^{*}\right) \rightarrow \mathrm{T}_{1}\left({ }^{3} \pi \pi^{*}\right)$ transition. Cui and Fang ${ }^{27}$ mapped the reaction paths and spin orbit coupling (SOC) matrix elements for the excited state relaxation of $2 \mathrm{tThy}$ in the gas phase using CASPT2. They found out three competitive ISC reaction paths through either $\mathrm{S}_{1}\left({ }^{1} \mathrm{n} \pi^{*}\right) \rightarrow$ $\mathrm{T}_{1}\left({ }^{3} \pi \pi^{*}\right), \mathrm{S}_{2}\left({ }^{1} \pi \pi^{*}\right) \rightarrow \mathrm{T}_{2}\left({ }^{3} \mathrm{n} \pi^{*}\right)$, or $\mathrm{S}_{2}\left({ }^{3} \pi \pi^{*}\right) \rightarrow \mathrm{T}_{3}\left({ }^{3} \mathrm{n} \pi^{*}\right)$ transitions. Gobbo and Borin, ${ }^{26}$ based on a similar approach, also reported multiple ISC pathways for $2 \mathrm{t}$ Ura in the gas phase, $S_{1}\left({ }^{1} n \pi^{*}\right) \rightarrow T_{1}\left({ }^{3} \pi \pi^{*}\right)$ and $S_{2}\left({ }^{1} \pi \pi^{*}\right) \rightarrow T_{2}\left({ }^{3} n \pi^{*}\right)$. Using explicit water solvation in CASPT2/MM simulations of 4tThd, Cui and Thiel ${ }^{25}$ determined three possible ISC paths for this system, $\mathrm{S}_{1}\left({ }^{1} \mathrm{n} \pi^{*}\right) \rightarrow \mathrm{T}_{1}\left({ }^{3} \pi \pi^{*}\right), \mathrm{S}_{2}\left({ }^{1} \pi \pi^{*}\right) \rightarrow \mathrm{T}_{2}\left({ }^{3} \mathrm{n} \pi^{*}\right)$, and $S_{2}\left(3 \pi \pi^{*}\right) \rightarrow T_{1}\left(3 \pi \pi^{*}\right)$, with the latter activated via vibronic couplings.

Pollum and Crespo-Hernandez, ${ }^{14}$ based on femtosecond broadband transient absorption measurements of 2t Thy and 2 tUra in water and acetonitrile, have more specifically proposed that the ultrafast ISC in these molecules takes place through the $S_{1}\left({ }^{1} n \pi^{*}\right) \rightarrow T_{1}\left(3 \pi \pi^{*}\right)$ pathway. Similar conclusion has been reached by Jiang et al.,22 through combined resonance Raman spectroscopy and wave packet propagation. This consensus, however, has been recently broken by Mai, Marquadt, and Gonzalez,, ${ }^{23}$ who, after carrying out a topographic analysis of the reaction pathways of $2 \mathrm{tUra}$ at CASPT2, have proposed that the ISC effectively occurs though a different reaction pathway, involving a $\mathrm{S}_{1}\left({ }^{1} \mathrm{n} \pi^{*}\right) \rightarrow \mathrm{T}_{2}\left({ }^{3} \pi \pi^{*}\right)$ transition.

In a couple of recent works, Pollum, Jockusch, and CrespoHernandez have experimentally studied systematic sequences of thio-substitution in thymine ${ }^{7}$ and uracil ${ }^{13}$ from the unsubstituted parent species, through the two possible single-substituted species, to the double-substituted species. They have shown that - as expected - either single or double thio-substitutions (Figure 2(a)) promotes a strong red-shift in the absorption spectrum, as well as increases the ISC rate and quantum yield. Less expectedly, however, they have also found out that thio-substitution in either position 2 or 4 leads to derivatives with fairly different absorption and ISC properties; with replacement at position 4 resulting in larger red-shifts, ISC rates, and quantum yields. Finally, they have shown ${ }^{7}$ that the absorptivity of 2substituted species is also dependent on whether the parent molecule is thymine or thymidine, with larger absorptivity in the latter. This finding, specially surprising as we may expect that the sugar group would play a minor role for the absorption, has also been observed before by Zhu and co-workers ${ }^{29}$ in the case of thymine.

In this work, we have applied multireference quantum chemical methods to systematically explore the photophysics of thymine and its thio-derivatives. Different from previous computational works in the field, our focus has not been on the determination of reaction pathways, but rather on the physical-chemical interpretation of a number of aspects in the photophysics of these systems, as a function of the number and position of the thio-substitutions.

We have first provided an extensive analysis of the absorption spectra. Based on these simulations, we have been able to determine the origin of the different redshift and absorptivity of each derivative. Moreover, we have also analyzed the SOC matrix elements between relevant states of the singlet and triplet manifolds. These quantities allowed us to explain the variations in the ISC rates of the diverse thio-derivatives.

Together, these results provide a clear picture of how thiosubstitutions impact absorption and ISC, helping to rationalize several previous experimental findings.

\section{COMPUTATIONAL DETAILS}

Kohn-Sham density functional theory (DFT) with the $\omega \mathrm{B} 97 \mathrm{XD}$ functional ${ }^{30}$ was used to optimize the ground state geometries of Thy, 2tThy, 4tThy, and 2,4dtThy. The thymidine derivative 2 tThd was optimized as well. Microsolvated (water) species were optimized too, as discussed later. All these calculations were based on the 6$311++\mathrm{G}(2 \mathrm{~d}, \mathrm{p})$ basis set ${ }^{31}$ and performed with Gaussian $09 .{ }^{32}$ Continuum solvent effects ${ }^{33}$ were considered in few cases, as discussed later. Cartesian coordinates of each optimized species can be found in the Supporting Information (SI, Section $\mathrm{S} 1$ ).

Excited states were computed with two multi-reference methods: the complete active space perturbation theory to the second order (CASPT2) in its multi-state (MS) version ${ }^{34}$ and with the combined density functional theory / multireference configuration interaction (DFT/MRCI) method, originally designed by Grimme and Waletzke. 35

For the MS-CASPT2 calculations, the active space was composed of 14 electrons in 10 orbitals $\left(2 n, 5 \pi, 3 \pi^{*}\right)$ using the ANO-RCC-VTZP basis set. ${ }^{36}$ For the absorption spectrum calculations, the CASSCF was averaged over 9 states. For the SOC matrix determination, independent calculations were done for the singlet and triplet manifolds, with the CASSCF averaged over 5 states in each one. Standard IPEA was globally adopted in the CASPT2 calculations. To deal with intruder states in the absorption spectrum, a o.1 a.u. imaginary shift was used. This shift was not needed for the computation of the SOC matrix. All MS-CASPT2 calculations were done with Molcas 8.34

DFT/MRCI is an effective multireference semiempirical approach to predict spectral properties of organic systems within $0.2 \mathrm{eV}$ accuracy. ${ }^{37}$ This method was used with the parameters derived in ref. 35 and with $E_{\text {sel }}=1$ hartree, based on Kohn-Sham orbitals computed with BHLYP functional. $3^{8}$ The calculations were carried out with the def2TZVP basis set ${ }^{39}$ and the corresponding auxiliary basis sets for the resolution-of-the-identity (RI) approach..$^{{ }^{\circ}} \mathrm{DFT}$ calculations were done with Turbomole, ${ }^{41}$ while the MRCI calculations were done with the original DFT/MRCI Hamiltonian, 35 using the program version developed by 
Grimme and Waletzke 35 and recently updated by Lyskov and co-workers. ${ }^{42}$

To provide a simple visualization of the absorption spectra, CASPT2 and DFT/MRCI vertical excitation energies and oscillator strengths have been convoluted with normalized Gaussian functions (o.23 eV standard deviation). Band envelops were also computed for specific cases using the nuclear ensemble approach. 43 In these simulations, 200 ground-state geometries were sampled according to a harmonic oscillator Wigner distribution and the excitation energies and oscillator strengths for each one were computed with time-dependent DFT (TDDFT), at B3LYP/6$311++G(2 d, p)$ level. The nuclear ensemble simulations were carried out with the Newton-X program 44,45 interfaced to Gaussian 09 .

Most of results discussed here are in the gas phase. We have decided for that after checking the effect of solvation, simulated via microsolvation plus continuum model, on the absorption spectrum. As we show in the SI (Section S2), this solvation scheme had little effect on the spectroscopic quantities and could not fully account for the shifts between experimental and computational results. However, as discussed in the next section, such shifts do not strongly affect data comparison and analysis.

SOC matrix elements were computed at CASPT2 level using an effective one-electron spin-orbit Hamiltonian based on atomic mean field integrals, as implemented in Molcas. ${ }^{46}$ These calculations were done at the $S_{1}$ state minimum of each molecule, which was optimized with TDDFT at $\omega$ B $97 \mathrm{XD} / 6-311++\mathrm{g}(2 \mathrm{~d}, \mathrm{p})$ level. The character of these stationary structures as surface minima was checked by normal mode calculations at the same level.

\section{RESULTS AND DISCUSSION}

\section{The absorption spectra}

Figure 2 shows experimental 7 (panel a) and calculated (MSCASPT2 (b) and DFT/MRCI (c)) absorption spectra of the four investigated species. The main features of the bright states contributing to these spectra are reported in Table 1. Tables including the dark states are given in the SI (Section $\mathrm{S}_{3}$ ). As explained in the Computational Details, the simulated spectra are simple Gaussian convolutions over the excited states, using energies and oscillator strengths at the ground state minimum. Three other features are worth noting: first, the simulated results are in the gas phase, while most of the experimental results are in solution; second, the simulated results are for thymine derivatives only, while the experimental results mix thymine and thymidine derivatives; third, the simulated results are vertical excitation wavelengths, while the experimental results are wavelengths at the band maxima.

In spite of these differences and approximations, it is rewarding that the simulated spectra reproduce all main spectroscopic features for each molecule, and they still render the right energy order among them. The main deviation between experiment and theory is in the description of the lowest band of Thy, 4tThy, and 2,4dtThy, whose simulations are fairly blue-shifted in comparison to the experiments. The excellent agreement between the computed and experimental 47 results for Thy in the gas phase makes clear that the main source of this blue shift is solvation. In particular, Li et al. ${ }^{8}$ have shown that computation of water solvation effects on a nucleobase, including solute-solvent interactions and environment state-specific polarization, may red-shift the absorbing $\pi \pi^{*}$ states as much as about $0.6 \mathrm{eV}$. Effectively, the shifts observed in the current results are smaller than that. For Thy, it is $0.4 \mathrm{eV}$ employing CASPT2 and o.6 eV employing DFT/MRCI (compared to the results in water). For 2 tThy, the shifts are smaller than $0.1 \mathrm{eV}$. For 4tThy, they are $0.3 \mathrm{eV}$ for both methods. Finally, for 2,4dtThy, they are smaller than $0.4 \mathrm{eV}$ for CASPT2 and smaller than $0.3 \mathrm{eV}$ for DFT/MRCI.

Experimental (see SI, Section $\mathrm{S}_{4}$ ) and computed oscillator strengths agree within 0.1 for most of transitions. However, there are some relevant differences between theory and experiment, especially for CASPT2, whose deviation may reach 0.44 . With DFT/MRCI, the largest deviation is 0.25 .

Taking the results from MS-CASPT2 as the reference, we can verify that DFT/MRCI calculations provide very similar excitation energies and oscillator strengths. The root mean square deviation between the two data sets is $0.14 \mathrm{eV}$ for the energy and 0.167 for the oscillator strength. The largest deviations occur for Thy, for which the first and fourth $\pi \pi^{*}$ excitations are $0.27 \mathrm{eV}$ higher in DFT/MRCI than in CASPT2.

To understand the relation between the thio-substitutions and the absorption spectra, we have analyzed the four lowest bright excitations for each molecule (Table 1). The coefficient of the main configuration for each state reveals that the spectra of Thy and its thio-derivatives can be simply reframed within a 4-electrons/4-orbitals minimum model (Scheme 1). These orbitals, as given by the KohnSham calculations used in the DFT/MRCI, are shown in Figure 3(a). The equivalent CASSCF orbitals used for $\mathrm{CASPT}_{2}$ are shown in the SI (Section $\mathrm{S}_{5}$ ). (We will base the following discussion on the Kohn-Sham orbitals because the configuration coefficients given in Table 1 are much closer to unity with DFT/MRCI than those with CASPT2.)

We have also performed a natural bond orbital (NBO) analysis 49 of the Kohn-Sham orbitals used in the DFT/MRCI, specially concerning the density distributions along the $\mathrm{C}=\mathrm{O} / \mathrm{S} \pi$-bond. These results are shown in Table S21 of the SI. The NBO fraction at the carbon atom in both $\mathrm{C}=\mathrm{O} \pi$ bonds of Thy is about 0.27 . After single sulfur replacement, this number increases to 0.61 at position 2 of 2tThy, but to just 0.32 in position 4 of 4 tThy. These results confirm that the carbon atoms in position 2 and 4 of Thy play different roles in the $\pi$-system and anticipates the distinct properties of the different thio-derivatives.

To visualize the relationship between the four excitations and the four MOs in the 4/4 minimum model, we draw a 
schematic diagram of the excitations for the four molecules in Figure 3(b) (MS-CASPT2) and Figure 3(c) (DFT/MRCI).

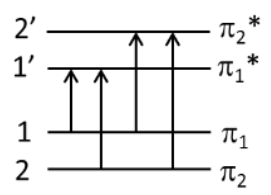

Scheme 1. 4/4 minimum model for $\pi \pi^{*}$ excitation of Thy and its thio-derivatives.

According to the experiments, the systematic thio-substitution of Thy and Thd has the following noticeable features in the absorption spectrum $^{7}$ (see Figure 2(a)):
1) Single or double substitutions lead to strong red shift in relation to the parent-unsubstituted species.

2) In the region above $250 \mathrm{~nm}, 2,4 \mathrm{dtThd}$ has a multistructured spectrum, while the other three species show a single band.

3) The red shift is significantly larger in 4 tThy than in 2tThy.

4) The absorptivity of $2 \mathrm{t}$ Thd is almost twice as large as that of 2tThy (not shown in Figure 2(a)).

Table 1. $\pi \pi^{*}$ excitations determining the absorption spectra of Thy, 2 tThy, 4 tThy, and $2,4 \mathrm{dtThy}$ in the gas phase according to MS-CASPT2 and DFT/MRCI. Experimental data in water, acetonitrile, and in the gas phase (brackets) are also shown. Values for Thd derivatives are given in parenthesis.

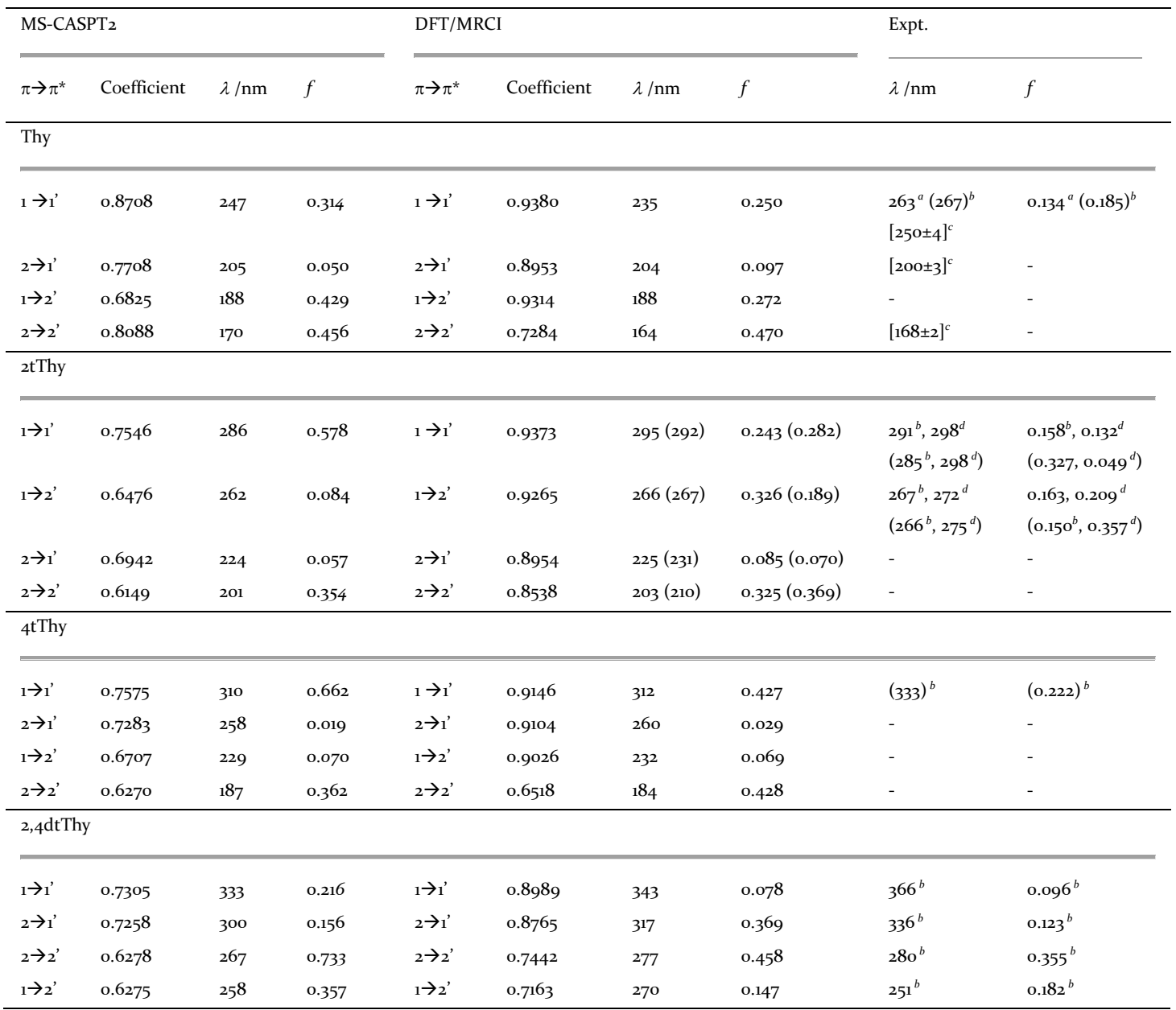

${ }^{a}$ Band maximum and band oscillator strength in water from ref. ${ }^{29} .{ }^{b}$ Band maxima and band oscillator strengths in water based on data of ref. ${ }^{7}$ (see SI, Section S4). ${ }^{c}$ Band maxima from electron energy loss spectroscopy of Thy in the gas phase, ref. ${ }^{47} .{ }^{d}$ Band maxima and oscillator strengths in acetonitrile from ref. ${ }^{15}$ (see $\mathrm{SI}$, Section $\mathrm{S}_{4}$ ).

Based on the spectrum calculations, we are already in position to address the first three questions. The fourth one will be discussed in the next section.
Concerning the first point, the overall red shift, it is simply caused by the well-known fact that $\mathrm{C}=\mathrm{S}$ bonds are weaker than $\mathrm{C}=\mathrm{O}$ bonds. This has already been observed in Ref.7. In the SI (Section S6), we use a simple Hückel model to 
show how the thio-substitution leads to a destabilization of the $\pi$ orbitals and stabilization of the $\pi^{*}$ orbitals, rendering smaller $\pi \pi^{*}$ transition energies than in the unsubstituted species.

Concerning the second point, the multi-structured spectra above $250 \mathrm{~nm}$, it is still consequence of the overall red shift in the thio-substituted species, which pushes up more transitions to longer wavelengths (see Figure $3(\mathrm{~b})$ ). In particular, for $2,4 \mathrm{dtThy}$, the structured high-energy band at about $270 \mathrm{~nm}$ appearing in the experiments (see Figure $\mathbf{2}(\mathrm{a})$ ) is due to the $\mathbf{2} \rightarrow \mathbf{2}^{\prime}$ and $\mathbf{1} \rightarrow \mathbf{2}^{\prime}$ transitions (Table $\mathbf{1}$ ).

Coming to the third point, the larger red shift in 4 tThy than in 2tThy, we note that there are two different effects going on. First, for the $1 \rightarrow 1^{\prime}$ and $2 \rightarrow 1$ ' transitions, the red shift of 4 tThy in relation to Thy is in fact larger than that of 2 tThy (see Figure $3(\mathrm{~b})$ ). However, for the $1 \rightarrow 2$ ' and $2 \rightarrow 2$ ' transitions, it is the opposite: the red shift is larger for 2tThy than for 4 tThy. Therefore, it is clear that the cause of the different red shifts in the two species should be searched in the nature of the $1^{\prime}$ and $2^{\prime} \pi^{*}$ orbitals.

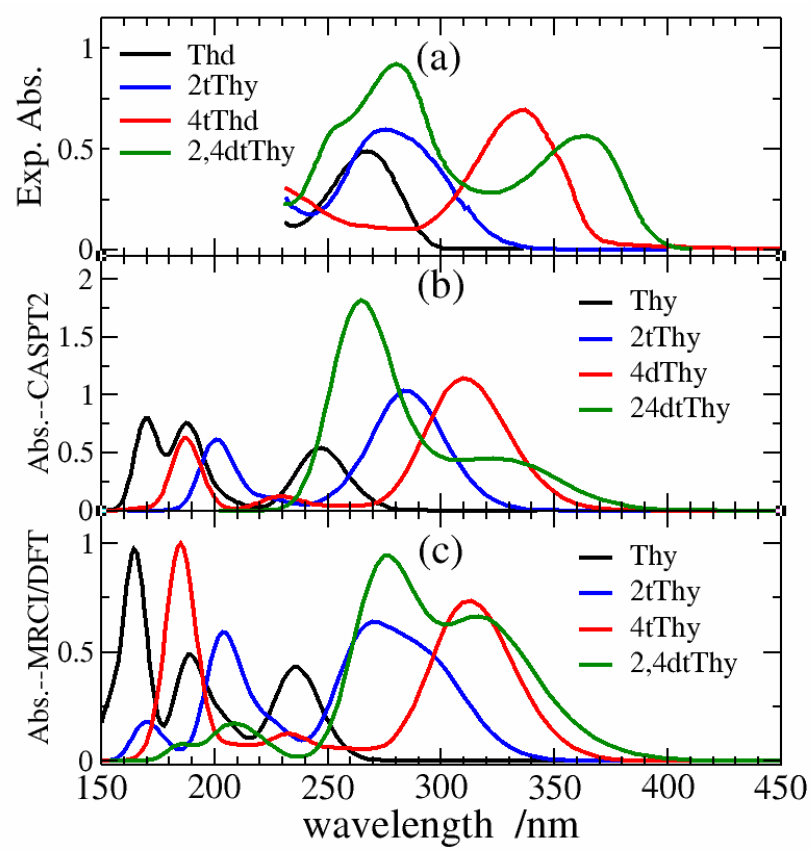

Figure 2. Absorption spectra of thymine and thymine derivatives: (a) experimental results in water from ref.7; (b) MSCASPT2 calculations; (c) DFT/MRCI calculations. Computational results in the gas phase.

Looking at these orbitals for 2Thy and 4Thy in Figure 3(a), we see that the electron density in the 1 ' orbital of 2 tThy extends to the sulfur atom, while the density in 1' of 4 tThy extends over the oxygen. Due to the same reason as discussed in point 1 above, the weaker $\mathrm{C}=\mathrm{S}$ bond as compared to $\mathrm{C}=\mathrm{O}$, a $\pi^{*}$ orbital along a CS bond is more stable than along a $\mathrm{CO}$ bond. As a consequence, excitations into $\mathrm{I}^{\prime}$ have larger energies in 2 tThy than in 4 tThy. On the other hand, the situation of the 2 ' orbitals is exactly the opposite: the electron density extends over the sulfur atom for 2 tThy and over the oxygen atom for 4 tThy. Thus, excitations into 2' have smaller energies in 2 tThy than in 4 tThy.

Thus, according to the analysis above, the different electron density distribution in the four main MOs involved in the $\pi \pi^{*}$ excitations is directly responsible for the observed shifts and band structure in the absorption spectra of thymine and its thio-derivatives.

(a)

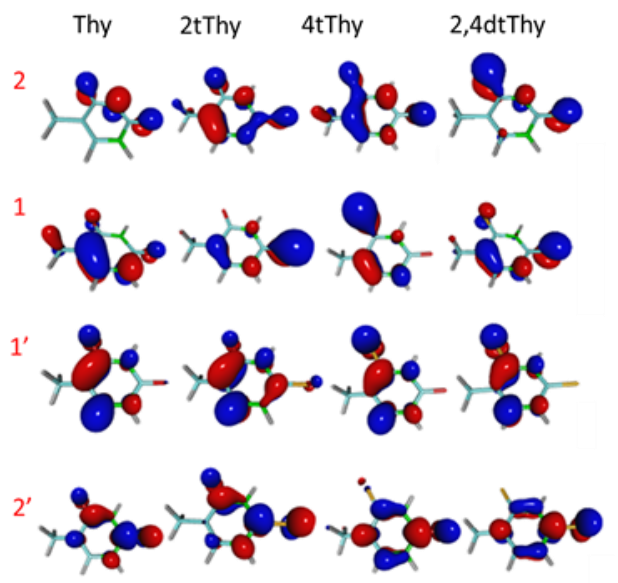

(b) DFT/MRCI

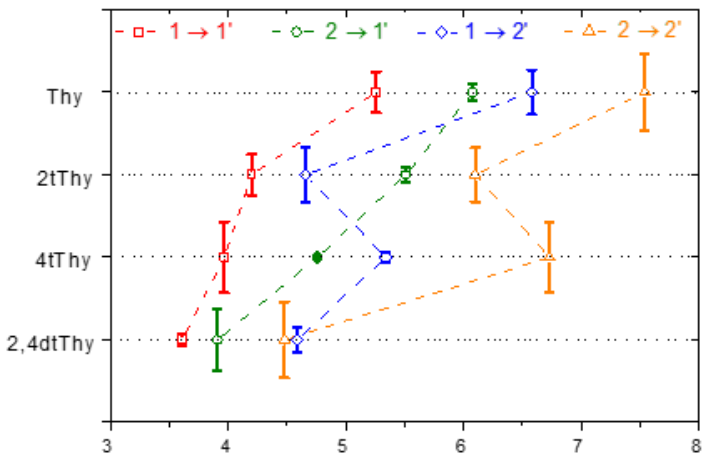

(c) MS-CASPT2

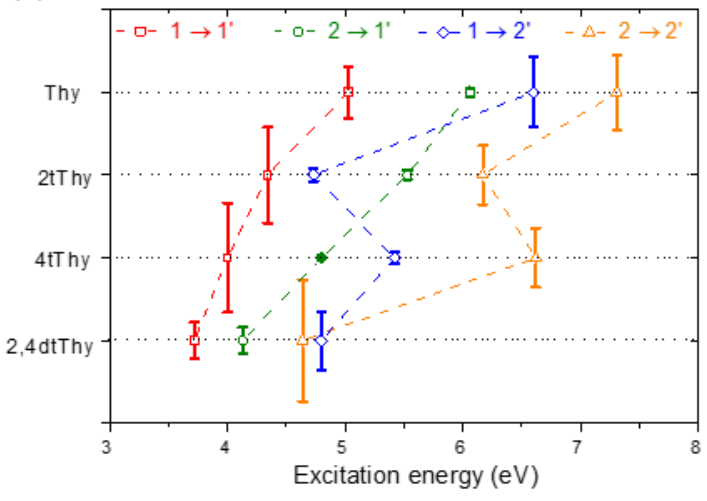

Figure 3. (a) The two occupied $\pi$ orbitals ( $M O_{1}$ and $\mathrm{MO}_{2}$ ) and unoccupied $\pi^{*}$ orbitals ( $\mathrm{MO} 1^{\prime}$ and $\mathrm{MO} 2^{\prime}$ ) involved in the excitations of Thy, 2tThy, 4tThy, and 2,4dtThy, from DFT calculations. (b) Comparison of different excitations including excitation energy and oscillator strength (given by the bars size) among the four molecules for DFT/MRCI. (c) The same as in (b) for MS-CASPT2. 


\section{Absorptivity difference in $2 \mathrm{tThy}$ and $2 \mathrm{tThd}$}

Another interesting phenomenon revealed by the experiments is that after glycosylation of 2 tThy, the formed 2t Thd still absorbs near $280 \mathrm{~nm}$, but its molar absorptivity significantly increases from $12 \times 10^{3}$ to about $20 \times 10^{3} \mathrm{M}^{-1} \mathrm{~cm}^{-1}$ (in water, $\mathrm{pH}$ 7.4). ${ }^{7}$ The combined oscillator strength of the first band raises from 0.321 in 2 tThy to 0.477 in 2 tThd, a ratio of 1.49 (Table 2 ). Zhu and co-workers ${ }^{29}$ have also reported a similar effect on the absorption spectrum of Thy and Thd in water, whose oscillator strength increased from 0.134 to 0.190 , a ratio of 1.42 . On the other hand, in acetonitrile, the increase in the molar absorptivity was more modest, from $12.4 \times 10^{3} \mathrm{M}^{-1} \mathrm{~cm}^{-1}$ at $290 \mathrm{~nm}$ for 2 tThy to $14.1 \times 10^{3} \mathrm{M}^{-}$ ${ }^{1} \mathrm{~cm}^{-1}$ at $285 \mathrm{~nm}$ for 2 tThd. ${ }^{15}$ The combined oscillator strength grows from 0.341 to 0.406 , a ratio of 1.19. Note yet that the molar absorptivity of 2 tThd in water $\left(\mathrm{pH}_{5.5}\right)$ is smaller than that reported in ref. ${ }^{7}, 17 \times 10^{3} \mathrm{M}^{-1} \mathrm{~cm}^{-1}$ at 276 nm. ${ }^{15}, 50$

Table 2. Total oscillator strength for the first absorption band of the nucleobase and of the corresponding nucleoside according to the experiments and to different computational models.

\begin{tabular}{lllll}
\hline & Model & $f_{\text {nucleobase }}$ & $f_{\text {nuceloside }}$ & Ratio \\
\hline Thy x Thd & & & & \\
\hline Expt. (ref. ${ }^{29}$ ) & & 0.134 & 0.190 & 1.42 \\
\hline Comp & DFT/MRCI & 0.249 & 0.315 & 1.26 \\
Comp. & TD-B3LYP & $0.128(0.178)^{a}$ & $0.194(0.228)$ & $1.52(1.28)$ \\
\hline 2tThy x 2tThd $^{2}$ & & & & \\
\hline Expt. (ref. ${ }^{7}$ ) & Water & 0.321 & 0.477 & 1.49 \\
Expt. (ref. ${ }^{15}$ ) & Acetonitrile & 0.341 & 0.406 & 1.19 \\
& DFT/MRCI & 0.569 & 0.474 & 0.833 \\
& TD-B3LYP & $0.376(0.571)$ & $0.329(0.415)$ & $0.88(0.73)$ \\
Comp. & TD-B3LYP; & $0.417(0.515)$ & $0.419(0.326)$ & $1.00(0.63)$ \\
& 6 waters + PCM & & & \\
Comp. & TD-B3LYP; & $0.422(0.493)$ & $0.321(0.391)$ & $0.76(0.79)$ \\
& band envelope & & & \\
\hline
\end{tabular}

${ }^{a}$ Values in parenthesis include solvent effects in the excited state calculations via PCM.

This absorptivity difference between the nucleobase and the deoxy-nucleoside may, in principle, be due to changes in the transition dipole moments caused by the glycosylation. This seems to be the case of Thd. As discussed in ref. ${ }^{29}$, the electronic density over one of the oxygen atoms of the ribosyl group is the key factor for the raise of the oscillator strength in the $1 \rightarrow 1$ ' transition dominating the first band of Thy and Thd. As shown in Table 2, the nucleoside/nucleobase oscillator strength ratio is in reasonable agreement with the experiment (1.26 at DFT/MRCI level).

Curiously, simple glycosylation cannot explain the absorptivity raise in the 2-thio-substituted species. The DFT/MRCI results show that the first band for the 2 tThy and 2 tThd overlaps the $1 \rightarrow 1^{\prime}$ and $1 \rightarrow 2$ ' transitions, with dominance of the latter (see Figure $3(\mathrm{~b})$ ). Like in the parent species, the oscillator strength for the $1 \rightarrow 1$ ' increases due to glycosylation, but that of the $1 \rightarrow 2$ ' decreases. The result, is a ratio below unity as shown in Table 2 (o.833 at DFT/MRCI level), directly diverging from the experimental ratios 1.49 in water or 1.19 in acetonitrile.

Discarding simple glycosylation as the source of the enhanced oscillator strength of 2 tThd, another possibility is that it is caused by specific solvent/chromophore interactions. Nevertheless, none of our tests including explicit micro-solvation plus continuum solvation could explain the oscillator strength increase. For instance, simulations of 2tThy and 2tThd including six explicit water molecules plus PCM render a ratio of only 0.63 (Table 2 ).

We also tested the vibrational distribution at the FranckCondon region. Using the nuclear ensemble approach based on TDDFT excitations, we have computed the band envelope for 2 tThy and 2 tThd in the gas phase and in water (PCM only). Again, the results for the ratio of the band areas were below unity, o.79 (Table 2).

Although we do not investigate it here, one last possibility to explain the absorptivity raise upon glycosylation is dimerization. Different from Thy, it is possible that the thiosubstitution in 2 tThy makes it more hydrophobic, leading to some degree of dimerization. 2tThd, however, should still not dimerize due to the high solubility of the sugar moiety. If this is the case, the experimental oscillator strength of 2 tThy would be underestimated, as the effective chromophore concentration would be smaller than that of the monomer (by up to $50 \%$ ).

At this point, however, we must admit that the larger absorptivity of 2 tThd as compared to that of 2 tThy is still an open question, with no clear and satisfactory explanation.

\section{Intersystem crossing}

As mentioned in the Introduction, thio-substitutions shift the triplet quantum yield of pyrimidine nucleobases from near zero to almost unity. ${ }^{7,15,18}$ The ISC lifetime, ${ }^{7,}{ }^{51}$ on its turn, ranges from 760 fs to 180 fs depending on the number and position of the thio-substitutions (see Table 3). Unsurprisingly, it is the longest for the unsubstituted species and the shortest for 2,4dtThy.

Following Kasha's rule, after the photoabsorption, we expect that all these systems quickly relax to the minimum of the singlet $S_{1}$ state, from where they may convert to the triplet manifold. Based on this scenario, we first optimized the $S_{1}$ state minimum of Thy, 2 tThy, 4tThy, and 2,4dtThy, and then computed the SOC matrix elements there. For effect of further analysis of the experimental and computational data, we stress that the computational simulations are in the gas phase, while the experimental results are in water. This difference certainly affects the energy gaps and state order, as the $\pi \pi^{*}$ and $n \pi^{*}$ react differently to protic solvation. ${ }^{48}$ To minimize this effect, we have additionally optimized the $S_{1}$ geometry in the continuum field of water as well, which had the general effect of stabilizing the $\pi \pi^{*}$ character, as expected.

The $S_{1}$ minimum geometries are presented in Figure 4. For Thy in water, the ring is strongly out-of-plane distorted 
and the $S_{1}$ state has $\pi \pi^{*}$ character. This is strikingly different from the result optimized in the gas phase, where the ring is planar and the $S_{1}$ state has $n \pi^{*}$ character. The thiosubstitution renders planar rings in both water and the gas phase. For 2tThy, however, the sulfur atom is displaced to out of plane, which has also been previously reported in ref. ${ }^{23}$. The $S_{1}$ minimum of 2 tThy strongly mixes $n \pi^{*}$ and $\pi \pi^{*}$ characters (the relevant molecular orbitals are shown in Section S8 of the SI). However, optimized in water, the $\pi \pi^{*}$ character increases, leading to a larger out-of-plane displacement of the sulfur atom than in the gas phase, where the $n \pi^{*}$ character predominates. For 4 tThy and $2,4 \mathrm{dtThy}$, the $S_{1}$ minimum, with pure $n \pi^{*}$ character, is fully planar and there are no remarkable differences between the optimized structures in either water or the gas phase.
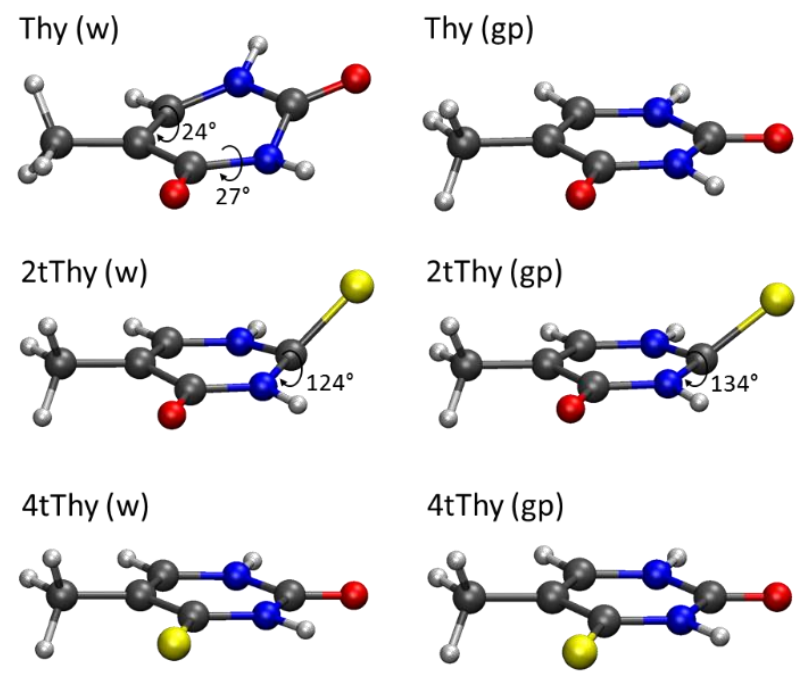

2,4dtThy (w)

\section{2,4dtThy (gp)}
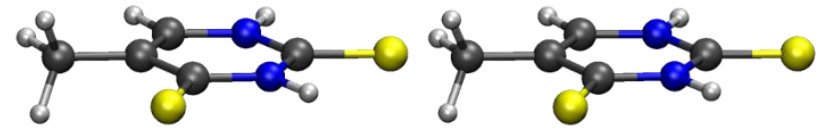

Figure 4. $\mathrm{S}_{1}$ optimized structures of Thy and its thio-derivatives in water ( $w$ ) and in the gas phase (gp).

The distribution of states at the $S_{1}$ minimum is shown in Figure 5 for geometries optimized in water (a) and in the gas phase (b). Based only on the energy gaps and on the ElSayed's rules, ${ }^{52}$ we can already draw the most probable scenario for ISC in these molecule. For Thy, with either $S_{1}$ minimum, singlet-triplet transfer should be very inefficient. For 2 tThy in water, the transfer may occur from $S_{1}$ into both $T_{1}$ and $T_{2}$, but in the gas phase it should happen only to $T_{1}$. For 4tThy and 2,4tThy, the transfer should occur from $S_{1}$ to $\mathrm{T}_{1}$ in both phases.

To investigate the relaxation after ISC, we also optimized the $T_{1}$ minimum structures at the same level of $S_{1}$ minima optimization for all four molecules. With these structures, we calculated singlet and triplet energies at CASPT2. (These energies are given in Tables S8-Sil of the SI.) As expected, the results confirm that energy $T_{1} / T_{2}$ energy gap increases after relaxation for all the molecules, implying the $\mathrm{T}_{1}$ state as the dominant one after ISC.

To go a step further in the ISC analysis, we have calculated SOC matrix elements between $S_{1}$ and the nearest triplets, $T_{1}$ and $T_{2}$ states at the $S_{1}$ optimized structures. The results are listed in Table 3. The SOC elements are given as the root of the sum of the square of the three hyperfine components:

$$
H_{S_{1} / T_{n}}^{S O}=\left[\sum_{m=-1,0,+1}\left(H_{S_{1} / T_{n, m}}^{S O}\right)^{2}\right]^{1 / 2} .
$$

(SOC elements for the thio-substituted Thd in the gas phase have been recently reported at TD-B3LYP level in ref. ${ }^{24}$. These values agree with ours within $20 \mathrm{~cm}^{-1}$. Hyperfine components are given in Section S8 of the SI.)

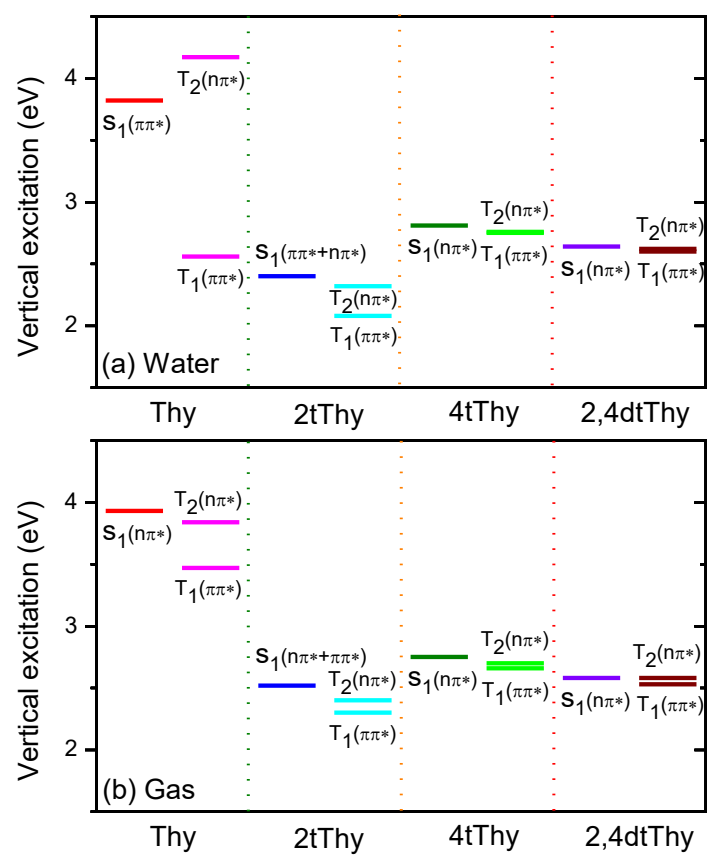

Figure 5. Singlet and triple state distributions at the $S_{1}$ minimum. CASPT2 using TDDFT geometries optimized in (a) water and (b) the gas phase.

Thy has very small SOC matrix elements, the largest being $22 \mathrm{~cm}^{-1}$ between $S_{1}$ and $T_{2}\left(S_{1}\right.$ minimum in water). For 2 tThy, the coupling increases by almost a factor 5 and the SOC elements are very similar for both triplet states, about 100 $\mathrm{cm}^{-1}$. At the $\mathrm{S}_{1}$ minimum in the gas phase, the SOC element increases to up $146 \mathrm{~cm}^{-1}\left(\mathrm{~S}_{1} / \mathrm{T}_{1}\right)$, which helps to explain why the ISC in acetonitrile is as faster as it is in water. ${ }^{15}$ For 4 tThy and 2,4dtThy, the SOC element between $\mathrm{S}_{1}$ and $\mathrm{T}_{1}$ increases in either phase to about $150 \mathrm{~cm}^{-1}$, while it is negligible between $\mathrm{S}_{1}$ and $\mathrm{T}_{2}$. 
The differences between SOC elements with $T_{1}$ and $T_{2}$ are related to how planar the $S_{1}$ minimum structure is. For 4tThy and 2,4dtThy, with planar structures (see Figure 4), the $n \pi^{*}$ and $\pi \pi^{*}$ states do not mix. In particular, as the $S_{1}$ state has $\mathrm{n} \pi^{*}$ character in these two molecules, it couples to the $T_{1}$ state, with $\pi \pi^{*}$ character, but not with the $T_{2}$ state, with $n \pi^{*}$ character. On the other hand, for the 2 tThy, whose sulfur atom is out of the plane, the $\mathrm{n}$ and $\pi$ systems strongly mix, rendering similar SOC strengths with $\mathrm{T}_{1}$ and $\mathrm{T}_{2}$. For Thy, using the structure optimized in water, although its $S_{1}$ minimum is also non-planar, it is still possible to assign a major $\pi \pi^{*}$ character to $S_{1}$ and $T_{1}$ and an $n \pi^{*}$ character to $\mathrm{T}_{2}$. Therefore, the SOC element is slightly larger for $S_{1} / T_{2}$ than for $S_{1} / T_{1}$. For the gas phase structure of Thy, the situation inverts: the $S_{1}$ state couples to $T_{1}$ but not to $T_{2}$.

In the simplest qualitative approach to relate the SOC elements to the ISC lifetime $\tau_{\mathrm{ISC}}$, these quantities are given in terms of the Golden Rule in the Condon approximation as: 53

$$
\frac{1}{\tau_{I S C}}=\frac{2 \pi}{\hbar} \sum_{n}\left(H_{S_{1} T_{n}}^{S O}\right)^{2} \rho_{E}
$$

where $\rho_{E}$ is the Franck-Condon weighted density of final vibronic states. It goes beyond the scope of this work to compute $\rho_{E}$ and in Table 3 we simply provide $\tau_{\mathrm{ISC}} \times \rho_{\mathrm{E}}$ to be compared to the experimental $\tau_{\text {Isc. }}$ (A full quantitative treatment of this problem for Thy can be found in refs. ${ }^{54}$, 55.) For each molecule, we have taken only the $S_{1} / T_{n}$ transition with the largest SOC element. In the case of 2 tThy, where both SOC elements have similar sizes, this approximation is justified by the much smaller energy gap in the $\mathrm{S}_{1} / \mathrm{T}_{2}$ transition than in the $\mathrm{S}_{1} / \mathrm{T}_{1}$.

The comparison between the computed $\tau_{\mathrm{ISC}} \times \rho_{E}$ and the experimental $\tau_{\text {ISC }}$ (Table 3 ) indicates that the reduction in the ISC lifetime from 2 t Thy to 4 tThy is caused by the increase in the SOC element from about 100 to about $150 \mathrm{~cm}^{-1}$.

Table 3. Total spin-orbit coupling elements, energy gaps, and state characters for $S_{1} / T_{1}$ and $S_{1} / T_{2}$ transitions for $S_{1}$ minimum geometries optimized in water and in the gas phase (in parenthesis). Estimates of the ISC time for transitions with largest SOC (multiplied by the density of states) and experimental ISC times.

\begin{tabular}{|c|c|c|c|c|c|c|c|c|}
\hline & \multicolumn{3}{|l|}{$\mathrm{S}_{1} / \mathrm{T}_{1}$} & \multicolumn{3}{|l|}{$\mathrm{S}_{1} / \mathrm{T}_{2}$} & \multirow{2}{*}{$\begin{array}{l}\tau_{\mathrm{ISC}} \times \rho_{E}\left(\mathrm{fs} . \mathrm{eV}^{-1}\right) \\
\text { compt }\end{array}$} & \multirow{2}{*}{$\begin{array}{l}\tau_{\text {ISC }}(\mathrm{fs}) \\
\text { expt }\end{array}$} \\
\hline & States & $\mathrm{H}^{\mathrm{SO}}\left(\mathrm{cm}^{-1}\right)$ & $\Delta E(\mathrm{eV})$ & States & $\mathrm{H}^{\mathrm{SO}}\left(\mathrm{cm}^{-1}\right)$ & $\Delta E(\mathrm{eV})$ & & \\
\hline Thy & $\begin{array}{l}{ }^{1} \pi \pi^{*} / 3 \pi \pi^{*} \\
\left({ }^{1} n \pi^{*} / 3 \pi \pi^{*}\right)\end{array}$ & $\begin{array}{l}1 \\
(49)\end{array}$ & $\begin{array}{l}-1.26 \\
(-0.46)\end{array}$ & $\begin{array}{l}{ }^{1} \pi \pi^{*} /{ }^{3} n \pi^{*} \\
\left({ }^{1} n p^{* / 3} n \pi^{*}\right)\end{array}$ & $\begin{array}{l}22 \\
\text { (o) }\end{array}$ & $\begin{array}{l}0.35 \\
(-0.09)\end{array}$ & 14081 & $760^{a, b}$ \\
\hline 2tThy & $\begin{array}{l}{ }^{1}\left(\pi \pi^{*}+\mathrm{n} \pi^{*}\right) / 3 \pi \pi^{*} \\
\left({ }^{1}\left(\mathrm{n} \pi^{*}+\pi \pi^{*}\right) / 3 \pi \pi^{*}\right)\end{array}$ & $\begin{array}{l}104 \\
(146)\end{array}$ & $\begin{array}{l}-0.32 \\
(-0.22)\end{array}$ & $\begin{array}{l}{ }^{1}\left(\pi \pi^{*}+\mathrm{n} \pi^{*}\right) /{ }^{3} \mathrm{n} \pi^{*} \\
\left({ }^{1}\left(\mathrm{n} \pi^{*}+\pi \pi^{*}\right) /{ }^{3} \mathrm{n} \pi^{*}\right)\end{array}$ & $\begin{array}{l}109 \\
(79)\end{array}$ & $\begin{array}{l}-0.08 \\
(-0.12)\end{array}$ & 574 & $620 \pm 60^{c}$ \\
\hline 4tThy & $\begin{array}{l}{ }^{1} \mathrm{n} \pi^{*} / 3 \pi \pi^{*} \\
\left({ }^{1} \mathrm{n} \pi^{*} / 3 \pi \pi^{*}\right)\end{array}$ & $\begin{array}{l}157 \\
(157)\end{array}$ & $\begin{array}{l}-0.06 \\
(-0.08)\end{array}$ & $\begin{array}{l}{ }^{1} \mathrm{n} \pi^{*} / 3^{3} \mathrm{n} \pi^{*} \\
\left({ }^{1} \mathrm{n} \pi^{*} /{ }^{3} \mathrm{n} \pi^{*}\right)\end{array}$ & $\begin{array}{l}5 \\
(25)\end{array}$ & $\begin{array}{l}-0.05 \\
(-0.04)\end{array}$ & 275 & $240 \pm \mathbf{2 0}{ }^{a, c}$ \\
\hline 2,4dtThy & $\begin{array}{l}{ }^{1} \mathrm{n} \pi^{*} / 3 \pi \pi^{*} \\
\left({ }^{1} \mathrm{n} \pi^{*} / 3 \pi \pi^{*}\right)\end{array}$ & $\begin{array}{l}152 \\
(153)\end{array}$ & $\begin{array}{l}-0.04 \\
(-0.06)\end{array}$ & $\begin{array}{l}{ }^{1} \mathrm{n} \pi^{*} / 3 \mathrm{n} \pi^{*} \\
\left({ }^{1} \mathrm{n} \pi^{*} /{ }^{3} \mathrm{n} \pi^{*}\right)\end{array}$ & $\begin{array}{l}2 \\
(1)\end{array}$ & $\begin{array}{l}-0.02 \\
(-0.01)\end{array}$ & 295 & $180 \pm 40^{c}$ \\
\hline
\end{tabular}

\footnotetext{
${ }^{a}$ Experimental results for the deoxy-nucleoside. ${ }^{b}$ Experimental ISC lifetime in water from ref. ${ }^{51}$. ${ }^{c}$ Experimental ISC lifetimes in water from ref. ${ }^{7}$.
} 


\section{CONCLUSION}

Aiming at rationalizing a series of recent experimental findings on the photophysics of thio-substituted thymines, we applied multireference computational methods to study thymine and a series of single and double thio-substituted species in terms of simulations of absorption spectra and computation of SOC matrix elements.

Photoabsorption spectra were determined at CASPT2 and DFT/MRCI levels for thymine, the single substituted 2tThy and 4tThy, and the double substituted 2,4dtThy. We have shown that different features experimentally observed in this series of molecules can be explained based on a simple 4-electrons/4-orbitals minimum model. The analysis of molecular orbitals involved in the excited states shows that the replacement of oxygen with sulfur in different positions has different effects on electron density delocalization and orbital energies, with direct consequences on the spectra.

We have also analyzed the significantly larger molar absorptivity of 2 tThd as compared to that of $2 \mathrm{tThy}$, revealed by steady spectroscopy. We tested a number of different factors that could be responsible for it, including the effect deoxyribose, interactions with solvent molecules, and the vibrational broadening at the Franck-Condon region. None of them, however, could provide a satisfactory explanation to the phenomenon. Although we raised the hypothesis that dimerization could be the underlying reason, we consider that the absorptivity raise of 2 tThy upon glycosylation is still an open question.

A qualitative analysis of the ISC process showed that the shortening of the ISC lifetime of 4 tThy as compared to that of 2 tThy in water is due to the larger SOC elements in the former molecule. Finally, the measured ISC lifetime for the unsubstituted thymine is at least 20 times faster than what is expected from the SOC elements. We have shown that a possible reason for this divergence may be a signal from $\mathrm{S}_{1}$ absorption overlapping the same spectral region used to assign the triplet states in the transient absorption spectra.

\section{ASSOCIATED CONTENT}

Supporting Information. Cartesian coordinates of optimized structures, solvent effects, details of CASPT2 and DFT/MRCI excitations, fitting of experimental oscillator strengths, Hückel analysis of red shift, SOC hyperfine components are given in supporting information. This material is available free of charge via the Internet at http://pubs.acs.org.

\section{AUTHOR INFORMATION}

\section{Corresponding Authors}

*SB: shuming.bai@univ-amu.fr; MB: mario.barbatti@univamu.fr

\section{Author Contributions}

The manuscript was written through contributions of all authors. All authors have given approval to the final version of the manuscript.

\section{ACKNOWLEDGMENT}

The authors thank support of the A*MIDEX grant ( ${ }^{\circ}$ ANR-11IDEX-0oo1-02) and of the project Equip@Meso (ANR-10EQPX-29-01), both funded by the French Government "Investissements d'Avenir" program. They are also in debt to Prof. Christel Marian, who kindly provided the latest version of the DFT/MRCI program.

\section{REFERENCES}

1. Barbatti, M. Borin, A. C. Ullrich, S., Photoinduced Processes in Nucleic Acids. Top. Curr. Chem. 2015, 355, 1-32.

2. Kleinermanns, K.; Nachtigallová, D.; de Vries, M. S., Excited State Dynamics of DNA Bases. Int. Rev. Phys. Chem. 2013, 32, 308-342.

3 . Douki, T., The Variety of Uv-Induced Pyrimidine Dimeric Photoproducts in DNA as Shown by Chromatographic Quantification Methods. Photochem. Photobiol. Sci. 2013, 12, 1286-1302.

4. Middleton, C. T.; de La Harpe, K.; Su, C.; Law, Y. K.; CrespoHernández, C. E.; Kohler, B., DNA Excited-State Dynamics: From Single Bases to the Double Helix. Annu. Rev. Phys. Chem. 2009, 60, 217-239.

5. Matsika, S., Modified Nucleobases. Top. Curr. Chem. 2015, $355,209-243$

6. Pollum, M.; Martínez-Fernández, L.; Crespo-Hernández, C. E., Photochemistry of Nucleic Acid Bases and Their Thio- and Aza-Analogues in Solution. Top. Curr. Chem. 2015, 355, 245-327.

7. Pollum, M.; Jockusch, S.; Crespo-Hernández, C. E., 2,4Dithiothymine as a Potent Uva Chemotherapeutic Agent. J. Am. Chem. Soc. 2014, 136, 17930-17933.

8. Zhang, X.; Jeffs, G.; Ren, X.; O’Donovan, P.; Montaner, B.; Perrett, C. M.; Karran, P.; Xu, Y.-Z., Novel DNA Lesions Generated by the Interaction between Therapeutic Thiopurines and Uva Light. DNA Repair 2007, 6, 344-354.

9. Brem, R.; Daehn, I.; Karran, P., Efficient DNA Interstrand Crosslinking by 6-Thioguanine and Uva Radiation. DNA Repair 2011, 10, 869-876.

10. Euvrard , S.; Kanitakis , J.; Claudy , A., Skin Cancers after Organ Transplantation. N. Engl. J. Med. 2003, 348, 1681-1691.

11. Massey, A.; Xu, Y.-Z.; Karran, P., Photoactivation of DNA Thiobases as a Potential Novel Therapeutic Option. Curr. Biol. 2001, 11, 1142-1146.

12. Reelfs, O.; Karran, P.; Young, A. R., 4-Thiothymidine Sensitization of DNA to Uva Offers Potential for a Novel Photochemotherapy. Photochem. Photobiol. Sci. 2012, 11, 148-154.

13. Pollum, M.; Jockusch, S.; Crespo-Hernandez, C. E., Increase in the Photoreactivity of Uracil Derivatives by Doubling Thionation. Phys. Chem. Chem. Phys. 2015, 17, 27851-27861.

14. Pollum, M.; Crespo-Hernández, C. E., Communication: The Dark Singlet State as a Doorway State in the Ultrafast and Efficient Intersystem Crossing Dynamics in 2-Thiothymine and 2-Thiouracil. $J$. Chem. Phys. 2014, 140, 071101.

15. Taras-Goślińska, K.; Burdziński, G.; Wenska, G., Relaxation of the $\mathrm{T}_{1}$ Excited State of 2-Thiothymine, Its Riboside and DeoxyribosideEnhanced Nonradiative Decay Rate Induced by Sugar Substituent. J. Photochem. Photobiol. A 2014, 275, 89-95.

16. Wenska, G.; Taras-Goslinska, K.; Lukaszewicz, A.; Burdzinski, G.; Koput, J.; Maciejewski, A., Mechanism and Dynamics of Intramolecular Triplet State Decay of 1-Propyl-4-Thiouracil and Its [Small Alpha]-Methyl-Substituted Derivatives Studied in Perfluoro-1,3Dimethylcyclohexane. Photochem. Photobiol. Sci. 2011, 10, 1294-1302.

17. Harada, Y.; Okabe, C.; Kobayashi, T.; Suzuki, T.; Ichimura, T.; Nishi, N.; Xu, Y.-Z., Ultrafast Intersystem Crossing of 4-Thiothymidine in Aqueous Solution. J. Phys. Chem. Lett. 2010, 1, 480-484.

18. Kuramochi, H.; Kobayashi, T.; Suzuki, T.; Ichimura, T., Excited-State Dynamics of 6-Aza-2-Thiothymine and 2-Thiothymine: Highly Efficient Intersystem Crossing and Singlet Oxygen Photosensitization. J. Phys. Chem. B 2010, 114, 8782-8789.

19. Reichardt, C.; Crespo-Hernández, C. E., Room-Temperature Phosphorescence of the DNA Monomer Analogue 4-Thiothymidine in Aqueous Solutions after Uva Excitation. J. Phys. Chem. Lett. 2010, 1, 22392243.

20. Harada, Y.; Suzuki, T.; Ichimura, T.; Xu, Y.-Z., Triplet Formation of 4-Thiothymidine and Its Photosensitization to Oxygen 
Studied by Time-Resolved Thermal Lensing Technique. J. Phys. Chem. B 2007, 111, 5518-5524.

21. Ruckenbauer, M.; Mai, S.; Marquetand, P.; González, L., Photoelectron Spectra of 2-Thiouracil, 4-Thiouracil, and 2,4-Dithiouracil. J. Chem. Phys. 2016, 144, 074303.

22. Jiang, J.; Zhang, T.-s.; Xue, J.-d.; Zheng, X.; Cui, G.; Fang, W.h., Short-Time Dynamics of 2-Thiouracil in the Light Absorbing S2(П $\pi *)$ State. J. Chem. Phys. 2015, 143, 175103.

23. Mai, S.; Marquetand, P.; González, L., A Static Picture of the Relaxation and Intersystem Crossing Mechanisms of Photoexcited 2Thiouracil. J. Phys. Chem. A 2015, 119, 9524-9533.

24. Pirillo, J.; De Simone, B. C.; Russo, N., Photophysical Properties Prediction of Selenium- and Tellurium-Substituted Thymidine as Potential Uva Chemotherapeutic Agents. Theor. Chem. Acc. 2016, 135, 15.

25. Cui, G.; Thiel, W., Intersystem Crossing Enables 4Thiothymidine to Act as a Photosensitizer in Photodynamic Therapy: An Ab Initio Qm/Mm Study. J. Phys. Chem. Lett. 2014, 5, 2682-2687.

26. Gobbo, J. P.; Borin, A. C., 2-Thiouracil Deactivation Pathways and Triplet States Population. Comput. Theor. Chem. 2014, 1040-1041, 195-201.

27. Cui, G.; Fang, W.-h., State-Specific Heavy-Atom Effect on Intersystem Crossing Processes in 2-Thiothymine: A Potential Photodynamic Therapy Photosensitizer. J. Chem. Phys. 2013, 138, 044315. 28. Gobbo, J. P.; Borin, A. C., On the Population of Triplet Excited States of 6-Aza-2-Thiothymine. J. Phys. Chem. A 2013, 117, 5589-5596.

29. Zhu, X.-M.; Wang, H.-g.; Zheng, X.; Phillips, D. L., Role of Ribose in the Initial Excited State Structural Dynamics of Thymidine in Water Solution: A Resonance Raman and Density Functional Theory Investigation. J. Phys. Chem. B 2008, 112, 15828-15836.

30. Chai, J.-D.; Head-Gordon, M., Long-Range Corrected Hybrid Density Functionals with Damped Atom-Atom Dispersion Corrections. Phys. Chem. Chem. Phys. 2008, 10, 6615-6620.

31. Hehre, W. J.; Ditchfield., R.; Pople, J. A., Self-Consistent Molecular-Orbital Methods.12. Further Extensions of Gaussian-Type Basis Sets for Use in Molecular-Orbital Studies of Organic-Molecules. J. Chem. Phys. 1972, 56, 2257-2261.

32. Chai, J.-D.; Head-Gordon, M., Systematic Optimization of Long-Range Corrected Hybrid Density Functionals. J. Chem. Phys. 2008, 128, 084106-15

33. Miertus, S.; Scrocco, E.; Tomasi, J., Electrostatic Interaction of a Solute with a Continuum - a Direct Utilization of Abinitio Molecular Potentials for the Prevision of Solvent Effects. Chem. Phys. 1981, 55, 117129.

34. Finley, J.; Malmqvist, P. A.; Roos, B. O.; Serrano-Andrés, L., The Multi-State Caspt2 Method. Chem. Phys. Lett. 1998, 288, 299-306.

35. Grimme, S.; Waletzke, M., A Combination of Kohn-Sham Density Functional Theory and Multi-Reference Configuration Interaction Methods. J. Chem. Phys. 1999, 111, 5645-5655.

$36 . \quad$ Roos, O. B.; Veryazov, V.; Widmark, P.-O., Relativistic Atomic Natural Orbital Type Basis Sets for the Alkaline and Alkaline-Earth Atoms Applied to the Ground-State Potentials for the Corresponding Dimers. Theor. Chem. Acc. 2003, 111, 345-351.

37. Silva-Junior, M. R.; Schreiber, M.; Sauer, S. P. A.; Thiel, W. Benchmarks for Electronically Excited States: Time-Dependent Density Functional Theory and Density Functional Theory Based Multireference Configuration Interaction. J. Chem. Phys. 2008, 129, 104103.

38. Becke, A. D., A New Mixing of Hartree--Fock and Local Density-Functional Theories. J. Chem. Phys. 1993, 98, 1372-1377.

39. Weigend, F.; Ahlrichs, R., Balanced Basis Sets of Split Valence, Triple Zeta Valence and Quadruple Zeta Valence Quality for $\mathrm{H}$ to $\mathrm{Rn}$ :
Design and Assessment of Accuracy. Phys. Chem. Chem. Phys. 2005, 7 3297-3305.

40. Weigend, F.; Häser, M.; Patzelt, H.; Ahlrichs, R., Ri-Mp2: Optimized Auxiliary Basis Sets and Demonstration of Efficiency. Chem. Phys. Lett. 1998, 294, 143-152.

41. Ahlrichs, R.; Bär, M.; Häser, M.; Horn, H.; Kölmel, C., Electronic-Structure Calculations on Workstation Computers - the Program System Turbomole. Chem. Phys. Lett. 1989, 162, 165-169.

42. Lyskov, I.; Kleinschmidt, M.; Marian, C. M., Redesign of the Dft/Mrci Hamiltonian. J. Chem. Phys. 2016, 144, 034104.

43. Crespo-Otero, R.; Barbatti, M., Spectrum Simulation and Decomposition with Nuclear Ensemble: Formal Derivation and Application to Benzene, Furan and 2-Phenylfuran. Theor. Chem. Acc. 2012, 131, 1237. 44. Barbatti, M.; Granucci, G.; Ruckenbauer, M.; Plasser, F.; Pittner, J.; Persico, M.; Lischka, H., NEWTON-X: a package for Newtonian dynamics close to the crossing seam, version 1.2, www.newtonx.org $\mathbf{2 0 1 1}$, access date: July 25, 2016.

45. Barbatti, M.; Ruckenbauer, M.; Plasser, F.; Pittner, J.; Granucci, G.; Persico, M.; Lischka, H., Newton-X: A Surface-Hopping Program for Nonadiabatic Molecular Dynamics. WIREs: Comp. Mol. Sci. 2014, 4, 2633

46. Malmqvist, P. Å; Roos, B. O.; Schimmelpfennig, B., The Restricted Active Space (Ras) State Interaction Approach with Spin-Orbit Coupling. Chem. Phys. Lett. 2002, 357, 230-240.

47. Abouaf, R.; Pommier, J.; Dunet, H., Electronic and Vibrational Excitation in Gas Phase Thymine and 5-Bromouracil by Electron Impact. Chem. Phys. Lett. 2003, 381, 486-494.

48. Li, Q.; Mennucci, B.; Robb, M. A.; Blancafort, L.; Curutchet, C., Polarizable Qm/Mm Multiconfiguration Self-Consistent Field Approach with State-Specific Corrections: Environment Effects on Cytosine Absorption Spectrum. J. Chem. Theory Comput. 2015, 11, 1674 1682.

49. Glendening, E. D.; Landis, C. R.; Weinhold, F., Natural Bond Orbital Methods. WIREs: Comp. Mol. Sci. 2012, 2, 1-42.

50. $\quad$ Faerber, P.; Scheit, K. H., Die Chemische Synthese Von 2.4 -

Dithio - Thymin - Nucleosiden. Chem. Ber. 1970, 103, 1307-1311.

51. Kwok, W.-M.; Ma, C.; Phillips, D. L., A Doorway State Leads to Photostability or Triplet Photodamage in Thymine DNA. J. Am. Chem. Soc. 2008, 130, 5131-5139.

52. El-Sayed, M. A., Spin-Orbit Coupling and the Radiationless Processes in Nitrogen Heterocyclics. J. Chem. Phys. 1963, 38, 2834-2838. 53. Marian, C. M., Spin-Orbit Coupling and Intersystem Crossing in Molecules. WIREs: Comp. Mol. Sci. 2012, 2, 187-203.

54. Etinski, M.; Fleig, T.; Marian, C. A., Intersystem Crossing and Characterization of Dark States in the Pyrimidine Nucleobases Uracil, Thymine, and 1-Methylthymine. J. Phys. Chem. A 2009, 113, 11809-11816 55. Etinski, M.; Tatchen, J.; Marian, C. M., Time-Dependent Approaches for the Calculation of Intersystem Crossing Rates. J. Chem. Phys. 2011, 134, 154105.

56. Hare, P. M.; Middleton, C. T.; Mertel, K. I.; Herbert, J. M.; Kohler, B., Time-Resolved Infrared Spectroscopy of the Lowest Triplet State of Thymine and Thymidine. Chem. Phys. 2008, 347, 383-392. 


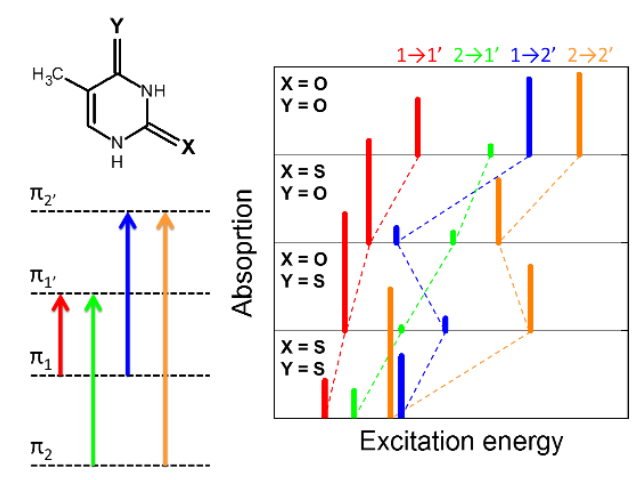

